\title{
Tabularia
}

\section{À l'origine du culte du Précieux Sang de Fécamp, le Saint Voult de Lucques}

On the origin of the cult of Holy Blood of Fecamp, the Holy Face of Lucca

Jean-Guy Gouttebroze

\section{OpenEdition}

Journals

Édition électronique

URL : http://journals.openedition.org/tabularia/1676

DOI : 10.4000/tabularia.1676

ISSN : 1630-7364

Éditeur :

CRAHAM - Centre Michel de Boüard, Presses universitaires de Caen

Référence électronique

Jean-Guy Gouttebroze, «À l'origine du culte du Précieux Sang de Fécamp, le Saint Voult de Lucques », Tabularia [En ligne], Guillaume de Volpiano : Fécamp et l'histoire normande, mis en ligne le 10 juillet 2002, consulté le 19 avril 2019. URL : http://journals.openedition.org/tabularia/1676 ; DOI : 10.4000/ tabularia.1676 


\title{
À l'origine du culte du Précieux Sang de Fécamp, le Saint Voult de Lucques. \\ On the origin of the cult of Holy Blood of Fecamp, the Holy Face of Lucca
}

\author{
Jean-Guy GOUTTEBROZE \\ UMR 6130, Université de Nice - Sophia Antipolis \\ gouttebr@unice.fr
}

Résumé:

Après la crucifixion, Nicodème, en compagnie de Joseph d'Arimathie, reçoit le corps du Christ et procède à son ensevelissement. Nicodème est un des derniers hommes à avoir eu un contact physique avec la dépouille mortelle du Christ - il l'a vue et touchée. De ce fait, il est à même de devenir un pourvoyeur de reliques. Il sculpte, inspiré par Dieu, le visage du Christ: cette œuvre, le Saint Voult, passe de Terre Sainte à Lucques. Dans une autre tradition, il recueille des particules ou des gouttes de sang christique qui, à la suite d'un périple miraculeux, seront portées, dans le tronc d'un figuier, jusqu'au rivage où doit s'élever l'abbaye de la Sainte-Trinité de Fécamp. Les deux corpus historico-légendaires ne sont pas indépendants l'un de l'autre. A certains indices, nous pouvons supposer que les clercs et les laïcs de Fécamp qui ont composé le récit de la translation du Précieux Sang, ont mis à contribution la tradition du Saint Voult de Lucques.

Mots-clés: crucifixion, Évangile, Fécamp, iconoclasme, image, incarnation, jongleurs, poèmes épiques, reliques, Sang (Précieux), Voult (Saint).

\begin{abstract}
:
After the Crucifixion, Nicodemus, accompanied by Joseph of Arimathaea, receives and buries the body of Christ. Nicodemus is one of the last people to have been in physical contact with Christ's mortal remains, which he saw and touched. Because of this he is in a position to become a provider of relics. Inspired by God he chissels the face of Christ. This sculpture, the Holy Face, travels from the Holy Land to Lucca. According to a second tradition, Nicodemus receives the 'particules' or drops of Holy Blood, which, following a miraculous journey, shall be carried inside the trunk of a fig tree to the coast where in due course the monastery of Holy Trinity at Fécamp will arise. The two semi-historical traditions are not independent of each other. Several aspects suggest that the clergy and laity at Fécamp responsible for the translation narrative of the Holy Blood may also have made a contribution to story of the Holy Face of Lucca.
\end{abstract}

Keywords: crucifixion, gospel, Fécamp, iconoclasm, image, incarnation, jongleurs, epic poetry, relics, Blood (Holy), Face (Holy). 
A quelques détails près, l'histoire légendaire du Précieux Sang de Fécamp prend sa forme définitive à la suite de la découverte, le 19 juillet 1171, dans les ruines de l'abbaye de la Sainte-Trinité, de deux étuis de plomb renfermant des particules du sang du Christ.

De cette tradition littéraire, il nous est parvenu un texte latin en prose et deux poèmes en octosyllabes rédigés en langue vernaculaire qui en sont une adaptation. Ces ouvres étaient destinées, pour leur instruction et leur édification, aux clercs et aux laïcs visitant le sanctuaire. Prévoyantes, les autorités monastiques avaient ménagé des moyens d'information qui tenaient compte des différents niveaux de culture.

Au demeurant, ces écrits qui ont été élaborés entre 1171 et 1210 environ ne sont pas le lieu d'une totale innovation. Depuis le début du XII ${ }^{\mathrm{e}}$ siècle circulait, du moins oralement, dans le cadre de l'abbaye, la notice historique d'une relique du sang du Christ, emmurée derrière une colonne de l'église abbatiale et qui guérissait malades et infirmes. Baudri de Bourgueil, qui avait rendu visite aux moines de la Sainte-Trinité entre 1107 et 1130, dans une lettre de remerciement à eux adressée, écrivait:

Le monastère se glorifie de posséder le sang de Notre Seigneur Jésus-Christ, inhumé par Nicodème, comme l'atteste saint Jean, sang qui a été recueilli sur ses membres: solennellement et en grand nombre, les pèlerins se pressent pour se rendre sur ce lieu ${ }^{1}$.

Cette référence à Nicodème devait être reprise et amplifiée par les anonymes qui, à partir de 1171, élaborèrent la tradition historico-légendaire du Précieux Sang, en y incluant des éléments d'origine locale.

Dans cette tradition, Nicodème s'impose comme un personnage clef. Des quatre évangélistes canoniques, seul saint Jean parle de lui: juif, pharisien, de rang social élevé, il a rejoint en secret les rangs des chrétiens. Associé à Joseph d'Arimathie, il procède à la mise au tombeau du Christ. Étant, avec Joseph d'Arimathie, le dernier homme à avoir tenu le corps du Christ, il deviendra, selon ses hagiographes, un pourvoyeur privilégié de reliques. Ce rôle sera d'autant plus reconnu que lui fut attribué un évangile apocryphe, en fait rédigé au $\mathrm{IV}^{\mathrm{e}}$ siècle et qui mentionne, de façon circonstanciée, ses activités de la Passion à la Résurrection.

En plaçant Nicodème à l'origine du périple qui devait amener la relique du Précieux Sang de la Palestine antique à Fécamp, les autorités de l'abbaye de la Sainte-Trinité n'innovaient pas: elles se référaient à Nicodème, car il était susceptible d'avoir recueilli le sang du Christ. Certes, une telle initiative pouvait provenir directement, comme un prolongement abusif, de l'Evangile de saint Jean. Il est cependant probable que, conjointement, les moines de Fécamp disposaient de textes

1. Baldrici, Itinerarium sive Epistola ad Fiscannenses, Patrologie Latine, CLXVI, Paris, Jacques-Paul Migne, 1894, col. 1182. Nous avons donné une traduction du texte latin de l'histoire légendaire du Précieux Sang, d'après l'édition de H. Omont De Inventione sanguinis Christi in GoUTTEBROZE, JeanGuy, Le Précieux Sang de Fécamp, origine et développement d'un mythe chrétien, Paris, Honoré Champion, 2000, p. 68-78. 
hagiographiques qui montraient comment Nicodème avait été, en se livrant à un travail de sculpteur, un propagateur de l'image du Christ. De nombreux sanctuaires, à cette époque, s'enorgueillissaient de posséder un crucifix qui portait une représentation, considérée comme fidèle, des traits du Christ: l'attribution de l'œuvre à Nicodème était une garantie de son authenticité. Citons, entre autres, les crucifix de Sainte Marie de Najère et de Rüe; mais, de toutes ces sculptures, la plus célèbre était le Saint Voult de Lucques, conservé et honoré dans la cathédrale Saint-Martin de cette ville. Ce qui fait du Saint Voult de Lucques un archétype des crucifix attribués à Nicodème, c'est l'ancienneté de son culte, qui semble avoir précédé tous les autres, et la littérature fournie dont il a été l'objet; c'est aussi la richesse et le rayonnement de la ville de Lucques qui ont fait connaître cette relique dans tout l'Occident chrétien.

Selon une tradition locale qui s'est développée à Lucques et qui est attribuée au chroniqueur Leboinus, c'est en 782, le premier vendredi après Pâques, que le Saint Voult est reçu dans cette ville. Des chrétiens l'avaient caché en Palestine afin de le soustraire aux atteintes des musulmans. Il fut trouvé à Ramla, dans la cave d'une demeure appartenant à un chrétien, Seleucus, par l'évêque Gualfredus qui était venu d'Italie. C'est à Ramla que Nicodème s'était retiré dès qu'il était devenu suspect à la communauté juive ${ }^{2}$. La date de 782 peut sembler bien précoce; ce n'est qu'au $\mathrm{X}^{\mathrm{e}}$ siècle que le Saint Voult est installé dans la cathédrale de Lucques et que s'élabore, à son sujet, une tradition littéraire étendue. Mais cette date n'a pas été choisie au hasard: c'est en effet à la fin du VIII siècle que s'intensifie, dans l'Occident chrétien, en réaction aux excès iconoclastes byzantins, le culte des images et que se mettent en place des procédures de récupération. De cette période date l'arrivée à Rome de l'image du Christ du Sancta Sanctorum; jetée à la mer par le prêtre Germannos qui voulait la sauver de la destruction, elle est alors portée miraculeusement sur les flots jusqu'à Rome, où le pape Grégoire II la recueille 3 . Cette image avait été, dit-on, confectionnée par Saint Luc et achevée par un ange. Dans les années ou les siècles qui ont suivi, sur ce modèle, s'est élaborée la légende du Saint Voult: saint Luc est remplacé par Nicodème; mais, selon Leboinus, l'intervention divine, qui se manifeste en la personne de l'ange, n'est pas totalement absente de cette reprise de la légende: Nicodème a sculpté « le très saint visage en mettant en œuvre un art qui n'était pas le sien, mais celui de Dieu ${ }^{4}$.

2. Voir sur ces points Almerico GUERRA, Notizie storiche del Volto Santo, Lucca, Tipografia arciv. s. Paolino, 1881, p. 36-40 et 53.

3. F. de MeLY, «L'image du Christ du Sancta Sanctorum et les reliques chrétiennes apportées par les flots ", Paris, Mémoires de la Société des Antiquaires de France, tome LVIII, 1902, p. 113-144. Le local du Sancta Sanctorum, ainsi nommé par référence au Saint des Saints du temple de Jérusalem, renferme, dans la basilique du Latran, les reliques les plus précieuses de la Chrétienté.

4. "Sacratissimum Vultum, non sua, sed divina arte disculpsit ", cité d'après Almerico GUERRA, Notizie storiche..., p. 16. 
Gervais de Tilbury, qui a voyagé en Italie à la fin du XII ${ }^{e}$ siècle, s'est peut-être rendu à Lucques et, certainement, sur le site de Luni. A Sarzana, il a pris en main et examiné l'ampoule du sang du Christ qui était jointe à la relique du Saint Voult. De cette reproduction même du Christ en croix, il donne, au chapitre XXIV de ses Otia imperialia, un aperçu de ses origines et de son histoire qui reprend la tradition qui avait cours localement ${ }^{5}$.

Toutefois, simultanément, le corpus légendaire du Saint Voult se dilate: au début du XIII ${ }^{e}$ siècle paraît un poème en langue d'oïl, de facture épique ${ }^{6}$. Il est composé en triptyque: découverte de la Croix, histoire du Saint Voult, miracle et martyr du jongleur Genois, en qui nous pouvons voir une adaptation de la figure de saint Genest. Dans ce texte, la légende primitive du Saint Voult est profondément remaniée. Nicodème et l'empereur de Constantinople David sculptent trois crucifix qu'ils jettent dans la mer de Grèce. Nicodème en a sculpté un, qui parviendra à Lucques; les deux autres, œuvres de David, iront l'un à Rome, l'autre à Rüe. Le Saint Voult est victime de son succès. Certes, seule la cathédrale de Lucques possède le crucifix le plus authentique, mais cela n'exclut pas que, sur les conseils de Nicodème, deux images du Christ en croix de même type ont été créées. L'église Saint-Vulphly de Rüe peut se recommander de la possession d'une de ces représentations.

A ces œuvres littéraires contribuant à la célébrité du culte du Saint Voult, il convient de joindre les allusions apparaissant ponctuellement dans les poèmes épiques des $\mathrm{XII}^{\mathrm{e}}$ et XIII ${ }^{\mathrm{e}}$ siècles. Elles connotent la dévotion particulière que les jongleurs - créateurs ou simples récitants des œuvres concernées - portent au Saint Voult. Citons, par ordre autant que possible chronologique, Aliscans (v. 4760-9), Ogier le Danois (II, laisse 367), Raoul de Cambrai (v. 4208-9).

En milieu anglo-normand, l'existence du Voult de Lucques était bien connue. Comme l'attestent successivement Guillaume de Malmesbury, Ordéric Vital et Wace, le roi Guillaume le Roux, qui meurt en 1100, jurait par le Voult de Lucques. Ses interlocuteurs, comme lui-même, connaissaient bien le sens de cette formule. Parmi eux, se trouvait son principal conseiller Herbert de Losinga, évêque de Norwich, ancien moine de Fécamp, qui continuait à entretenir des relations avec son monastère d'origine. Il serait étonnant que les moines de Fécamp n'aient pas compris ce qu'un ministre, issu de leurs rangs, était appelé à comprendre. En outre, l'empereur Henri V est un protecteur du sanctuaire de Lucques qu'il visite en 1100. Quelques années plus tard, il signe un diplôme qui confirme les privilèges de la cathédrale? ${ }^{7}$ Or, Henri V est un allié d’Henri I ${ }^{\text {er }}$ Beauclerc, dont il épouse

5. Gervais de Tilbury, Otia imperialia, in Scriptores Rerum Brunswicensium, éd. Leibniz, Hanovre, 1707, p. 967-8.

6. Le Saint Vou de Luques, éd. W. Foerster, Mélanges Chabaneau, Romanische Forschungen, Erlangen, Verlag von Fr. Junge, 1907.

7. Almerico GUERRA, Notizie storiche..., p. 104 
la fille en 1114: de nouveau, la dynastie anglaise a l'occasion d'être renseignée sur le Saint Voult.

Plus généralement, Lucques était un des lieux les plus réputés pour la fabrication des tissus de soie utilisés à la fois par l'aristocratie et, surtout, par l'Eglise. Les marchands lucquois qui se déplaçaient dans toute l'Europe ont certainement diffusé les vertus attachées au pèlerinage de Lucques, l'éclat des cérémonies qui s’y déroulaient, les miracles produits par le Saint Voult.

Le souvenir du Saint Voult à Fécamp est aussi bien présent pour des raisons théologiques.

C'est l'Iconoclasme qui, à la fin du VII e siècle, provoque l'exportation, depuis l'empire byzantin vers la chrétienté occidentale, d'images du Christ, de la Vierge et des saints. La tradition légendaire relative au Voult de Lucques est tributaire d'un mouvement iconodoule qui s'était établi dans la partie latine de la chrétienté. L'Incarnation justifiait la représentation de Dieu fait Homme et de ceux qui avaient participé à son activité terrestre. Elle rendait caducs, sur ce point, les interdits de l'Ancien Testament ${ }^{8}$. Comme l'image du Sancta Sanctorum, la représentation du Christ en croix attribuée à Nicodème s'affirmait comme un manifeste anti-iconoclaste.

Les jongleurs - musiciens, chanteurs, récitants, compositeurs, mimes - dont nous avons souligné l'attachement au Saint Voult, sont des gens du spectacle, donc de la représentation. Leurs activités sont condamnées par les autorités ecclésiastiques, comme frivoles, obscènes, dégradantes. Seuls trouvent grâce auprès du clergé ceux qui chantent les exploits des héros épiques et chrétiens et, dans le cadre même de l'abbaye de Fécamp, les musiciens et les chanteurs. Si nous en croyons le texte d'un vidimus de 1402, ceux-ci ont été organisés en confrérie dès l'époque de Richard II et de Guillaume de Volpiano, et cette organisation fut renouvelée sous les abbatiats d'Henri de Sully et de Raoul d'Argences ${ }^{9}$. Par l'intermédiaire des jongleurs, la tradition du Saint Voult dont ils étaient porteurs avait connu un regain de notoriété. Participant aux activités médiatiques de l'abbaye, ils ont vraisemblablement été sollicités pour diffuser la légende du Précieux Sang; peut-être ont-ils participé à sa composition, si ce n'est directement, du moins en informant et en influençant les membres de la collectivité monastique.

Le poème du Saint Voult de Lucques en langue vernaculaire, édité par W. Foerster, est une œuvre de jongleur. Sa troisième partie constitue une apologie, face au mépris et à l'avarice du clergé, des mérites d'un chanteur qui s'accompagne de sa vielle. Un texte latin du XII ${ }^{e}$ siècle, conservé à Lucques et formant une annexe au récit de Leboinus, racontait aussi comment un ménestrel, honteux de ne pouvoir faire une offrande au Saint Voult, se mettait à chanter devant lui en

8. Exode, 20, 4, Deutéronome, 5, 8.

9. Sur ce point, voir Jean-Guy GoutTebroze, Le Précieux Sang..., p. 39-49. 
son honneur, en s'accompagnant d'un instrument qui était vraisemblablement une vielle. Pour le récompenser, le Saint Voult avait projeté sur sa poitrine, in gremium, un de ses souliers d'argent.

L'expression de la bienveillance divine envers les jongleurs est encore plus nettement marquée dans le poème du Saint Voult en langue vernaculaire. Ce texte du début du XIII ${ }^{e}$ siècle, dont la langue est proche de celle qui devait être parlée non loin de Fécamp, relève de leur répertoire. Notons d'abord une sorte de signature: quand Hélène est invitée par son époux David à se retirer d'un combat, elle refuse, disant: "Ne nus jongleres mauvaisement n'en chant» (v. 227). C'est un motif traditionnel de la création épique dont les jongleurs sont, par excellence, les propagateurs. Par ailleurs, le poème emprunte sa forme à l'épopée: une succession de laisses assonancées en décasyllabes.

Cette œuvre, qui reprend la tradition latine du Saint Voult, s'en éloigne en plusieurs endroits, et ces écarts sont révélateurs, car ils signalent les mouvements d'une inspiration originale. Le Saint Voult est envisagé en lui-même; en effet, il n'est fait nulle mention de l'ampoule ou des ampoules de sang du Christ dont il est porteur. Sa sainteté et sa signification propres s'en trouvent accrues. Ce n'est plus dans une barque soigneusement calfeutrée qu'il vogue vers l'Italie. Les personnages jettent des crucifix dans la mer, les confiant au soin de la Providence. Certes, ce comportement s'explique par la finalité et la destinée des reliques: il est nécessaire qu'elles parviennent respectivement à Lucques, à Rome et à Rüe. Mais l'indication de la mer de Grèce, donc du littoral byzantin, alors que David et Nicodème se trouvent en Terre Sainte, renvoie à la persécution iconoclaste: ils réitèrent le geste du prêtre Germannos.

Il n'est pas fortuit que se soit établie, en l'occurrence, une relation entre le thème du Saint Voult et la condition de jongleur. Il existe entre ces deux entités un rapport logique. Le jongleur est un support de représentations - musicales, chantées, récitées, composées, dramatisées -; il peut, à ce titre, revendiquer sa dignité que le clergé met en cause. En revenant à l'origine de la dispersion des images du Christ en agonie, il produit une explication et une justification de sa condition.

Le culte des images peut être tempéré: il est un moyen de commémoration et de méditation. A ce niveau déjà, se trouve légitimée la fonction du jongleur. Mais il peut être beaucoup plus radical quand l'image est investie des vertus mêmes de la personne qu'elle représente. Elle devient un facteur de médiation. Les auteurs de la légende du jongleur Genois vont jusque là: alors qu'il chante devant le Saint Voult, le saint Esprit vient animer la sculpture:

Devant le Vous commencha a chanter,

Li sains Espirs commenche à avaler

Qui le Vous fait parler et remüer (v. 439-41)

Il s'en retire quand le jongleur a obtenu satisfaction des autorités ecclésiastiques: 


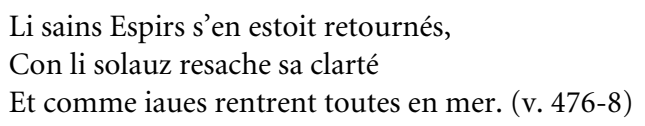

En associant, en des termes aussi précis, ce miracle à l'activité d'un jongleur, le texte pose le problème de la transcendance des images, de leur pouvoir médiatique et du statut de ceux qui participent à l'élaboration des représentations. La présence des jongleurs à Fécamp appelle la référence au Saint Voult et, réciproquement, les références littéraires à la relique lucquoise impliquent dans bien des cas la participation des jongleurs. Leur action, en synergie avec celle des moines bénédictins a présidé à la composition de la légende du Précieux Sang.

Ce faisceau de renseignements historiques et idéologiques convergents montre avec certitude que la communauté religieuse de Fécamp, lorsqu'elle s'apprête à donner une histoire définitive à la relique du Précieux Sang, connaît bien celle du Saint Voult de Lucques.

Que le corpus historico-légendaire ait été déjà bien élaboré, alors que la relique était encore dissimulée dans une colonne murale du sanctuaire, ou qu'il se soit formé après que cette relique eut été dégagée, la recherche d'une structure narrative qui conduisait à la Passion et, par là, à l'activité de Nicodème, découvrait comme modèle la légende du Saint Voult.

Le sang du Christ, en effet, n'est pas absent du crucifix que les habitants de Lucques et ceux de Luni découvrent sur le rivage. Comme beaucoup de crucifix miraculeux, l'image du Saint Voult porte, selon les traditions, une ou deux ampoules de sang christique. Dans un monde germanisé, il est possible aussi qu'une confusion entre vult et blutt ait orienté l'attention d'auditeurs qui pressentirent, sous le visage, la présence du sang. Ce phénomène d'attraction linguistique, sous une forme différente, s'exerce aussi à Rüe où l'église de saint Vulphly - vraisemblablement à l'origine un saint Loup - accueille un crucifix sur lequel figure le Saint Voult.

La nécessité d'expliquer l'origine d'un culte du sang du Christ qui, en Normandie, pouvait seulement être l'effet d'une translation, d'un périple miraculeux, trouvait, dans la navigation du Saint Voult, un précédent intéressant. Toutefois, la barque étanche qui portait le Saint Voult devint tronc de figuier, parce que préexistait au culte du Précieux Sang un culte populaire et païen de l'arbre et qu'un phénomène de fausse étymologie faisait de Fécamp, Fiscannum, lieu riche en poissons, une plaine, un champ du figuier, fici campus ${ }^{10}$.

La légende du Saint Voult présente donc des principes de fonctionnement que les conteurs de Fécamp, clercs et laïcs confondus, vont adapter à une réalité locale qui trouve un nouveau sens, sans perdre totalement son originalité.

Quant au texte qui naît, il se ressent, par endroits, de cette imitation. Le point principal qui dénonce le phénomène de réemploi est le geste de Nicodème raclant

10. Ibid., p. 82-95. 
de son couteau le corps du Christ pour en détacher des morceaux de sang coagulé qui y adhèrent. Cette initiative brutale ne peut s'expliquer que par le geste du sculpteur sur bois ayant formé le Saint Voult. Les moines de Fécamp auraient pu retenir l'image d'une réception, plus noble, celle du sang du Christ au pied de la croix.

Peut-être ont-ils adapté le motif du transport de la relique du Saint Voult sur un char tiré par des bœufs. L'évêque de Lucques, Jean, voulant neutraliser les prétentions des habitants de Luni à retenir chez eux le Saint Voult, le fait placer sur un char, escomptant que la Providence déciderait de la direction vers laquelle s'orienterait la relique. L'attelage s'engagea sur le chemin de Lucques. Le tronc du figuier qui contient la relique du Précieux Sang connaît également un transport miraculeux. Alors qu'il est impossible de l'arracher de la terre pour le hisser sur un chariot, un mystérieux personnage va, sans peine, s'acquitter de cette tâche. Dieu conduit alors l'attelage là où devait s'élever l'église de la Sainte-Trinité. A cet endroit, le tronc prend un tel poids qu'il écrase le chariot. Le pèlerin, avant de disparaitre, recouvre le tronc d'un tas de pierre. L'imitation semble évidente; toutefois, comme les transports d'objets rituels, de statues de divinité sur des chars appartiennent aussi à un patrimoine mythologique général, il n'est pas exclu que, sur ce point, nous soyons en présence d'une tradition locale ravivée par la légende du Saint Voult. Signalons enfin que l'homme à qui Nicodème, à la fin de sa vie, confie le Saint Voult est un de ses amis, Isaccar, personnage qui, dans la tradition du Précieux Sang, deviendra son neveu, Isaac.

Il est évident que le corpus historico-légendaire du Saint Voult de Lucques précède chronologiquement l'établissement du corpus qui, à Fécamp, lui correspond. Il y a bien, du premier au second, un processus de reprise et d'imitation. Toutefois, ce mouvement est limité. Les clercs et les laïcs de Fécamp qui s'intéressaient au passé de l'abbaye disposaient en effet de moyens qui leur permettraient de s'affranchir de cette tutelle, à savoir le modèle que fournissaient, par ailleurs, d'autres récits de translation, d'Orient en Occident, de la relique du sang du Christ et vraisemblablement, sur place, la préexistence d'un culte de l'arbre qui se renouvelle dans le motif du tronc du figuier. La tradition du Précieux Sang, qui s'impose comme syncrétique, tire en partie son originalité d'éléments locaux. Ainsi se trouvaient ménagées la persistance d'une pratique indigène et son adaptation au monde chrétien. D'où le maintien d'un potentiel de sacralité qu'une procédure d'imitation totale aurait émoussé ou même, anéanti. Un grand sanctuaire, alors qu'un modèle célèbre appelait les pèlerins, ne pouvait se contenter d'une reproduction. 